\title{
CAM WITH SPECIAL SPLINES FOR SOLVING OF DIFFUSION-CONVECTION PROBLEMS WITH DISCONTINUOUS COEFFICIENTS FOR LAYERED MATERIALS EXPOSED TO FIRE
}

\author{
Aivars Aboltins ${ }^{1}$, Harijs Kalis ${ }^{2}$, Ilmars Kangro ${ }^{3}$ \\ ${ }^{1}$ Latvia University of Life Sciences and Technologies, Latvia; \\ ${ }^{2}$ Institute of Mathematics and Computer Science, University of Latvia, Latvia; \\ ${ }^{3}$ Rezekne Academy of Technologies, Latvia \\ aivars.aboltins@inbox.lv, harijs.kalis@lanet.lv, ilmars.kangro@rta.lv
}

\begin{abstract}
In this paper we consider the conservative averaging method (CAM) for solving the diffusionconvection initial -boundary value problem (IBVP) with piece-wise coefficients in two layered domains. The created special exponential and hyperbolic type approximation functions - splines interpolate the middle integral values of the piece-wise smooth function, and thus the studied IBVP is reduced to the problem of the system of ordinary differential equations. The solution of the corresponding 1-D IBVP with diffusion was obtained also numerically, using the Matlab routine "pdepe" and the numerical solution was compared with the analytical solution. The 1-D diffusion-convection and 1-D diffusion boundary problems were solved and the solutions obtained were analysed with CAM (in case of exponential and hyperbolic splines) and also with Matlab "pdedpe" programme. Higher accuracy of the calculations in solving the 1-D diffusion-convection IBVP (case of discontinuous coefficients with a "jump-off" effect of the solution of IBVP) was identified by CAM. A practical task was solved: the temperature was calculated through the two layered material of gypsum products exposed to fire and the calculations were compared with the results obtained at the Faculty of Environment and Civil Engineering of the Latvia University of Life Sciences and Technologies. The data processing and analysis tools MS Excel and IBM SPSS Statistics20 tested the coincidence of the numerical calculations performed with the above mentioned experimental results.
\end{abstract}

Keywords: conservative averaging method, exponential splines, gypsum products, heat distribution, hyperbolic splines.

\section{Introduction}

The initial boundary value problems (IBVP) described by PDE with piece-wise coefficients in multi-layered domains are currently the subject of studies.

The interest is often caused by problems in themselves, but even more interesting are their solutions: mainly numerical ones, because analytical solutions can only be obtained in the ordinary sense (without changing the number of dimensions of the boundary-value problem) in the simplest cases.

The article deals with a universal method for solving the second order of partial differential equations - consider the conservative averaging method (CAM), the essence of which is a reduction in the number of dimensions of a given IBVP, with a view to obtaining analytical expressions (formulas) of the solution. The further solution of the IBVP includes a repeated reduction in the number of dimensions or applying of numerical methods to solve the acquired IBVP.

The unknown function is replaced by the approximated solution - the special spline with two different functions, which interpolate the middle integral values of the piece-wise smooth function.

The functions of the exponential and hyperbolic type spline are created and used with parameters that have to be chosen in the appropriate way to decrease the error of approximation of the solution.

It should be noted that, in a limited case, when the parameters of the spline function tend to zero, we have the integral parabolic spline, obtained from A.Buikis [1]. The solving of 3-D IBVP in $\mathrm{N}$ layer domain is considered in [2].

The solving of 1-D diffusion-convection IBVP using CAM and also Matlab routine "pdepe" is studied in the present article. CAM makes it possible to solve the IBVP with discontinuous convection coefficients. In the paper [3] the convection-diffusion problem with discontinuous coefficients is approximated by the implicit finite-difference scheme. Different cases of convection coefficients were chosen in numerical experiments to investigate the impact of the "jump-off" effect of the IBVP solution. 
As an application for modelling of transfer processes we study the heat transfer processes through layered material of gypsum products (gypsum foam and gypsum board) exposed to fire.

Research on gypsum materials as good building and finishing materials is important in various aspects, but from a fire safety point of view, it is important to study the properties of these materials directly in processes exposed these materials to fire.

The heat conductivity and transfer properties of gypsum materials, their thermal persistence and their general characteristics during the heating and burning processes have been studied in [4-6].

There are often experimental difficulties in temperature determining when heating gypsum material, so mathematical modelling for temperature calculation without direct measurement is very useful, of course, provided that the mathematical model created is sufficiently accurate for engineering calculations.

Therefore, a mathematical model for solving the 1-D IBVP was created, where the temperature on two layers of gypsum material was calculated, taking into account the dependence of the layers various density and diffusion coefficients on the burning temperature.

The data processing tools MS Excel and IBM SPSS Statistics20 were used to verify the accuracy of the mathematical model with the Paired-Comparison test.

\section{Materials and methods}

\section{The mathematical model}

The nonstationary diffusion- convection problem is studied in 1-D domain $\Omega=\left\{(z): 0 \leq z \leq L_{z}\right\}$, which consists of $N$-layered medium $\Omega_{i}=\left\{(z): z \in\left(z_{i}-z_{i-1}\right)\right\}, i=1^{-}, N$, where $H_{i}=z_{i}-z_{i-1}$ is the height of layer $\Omega_{i}, z_{0}=0, z_{N}=L_{Z}$. We must calculate the distribution of concentrations $u_{i}=u_{i}(z, t)$ in every layer $\Omega_{i}$ at the point $(z)=\Omega_{i}$ and at the time $t$ by solving the following IBVP for partial differential equation (PDE):

$$
\left\{\begin{array}{l}
\frac{\partial u_{i}}{\partial t}=\frac{\partial}{\partial z}\left(D_{i z} \frac{\partial u_{i}}{\partial z}+r_{i z} u_{i}\right)-a_{i 0}^{2} u_{i}+F_{i}, z \in\left(z_{i-1}, z_{i}\right), t \in\left(0, t_{f}\right), i=\overline{1,2}, \\
D_{1 z} \frac{\partial u_{1}(0, t)}{\partial z}-\beta_{z}\left(u_{1}(0, t)-C_{0 z}\right)=0, \\
D_{N z} \frac{\partial u_{N}\left(L_{z}, t\right)}{\partial z}+\alpha_{z}\left(u_{N}\left(L_{z}, t\right)-C_{a z}\right)=0, \\
u_{i}\left(z_{i}, t\right)=u_{i+1}\left(z_{i}, t\right) q_{i}, D_{i z} \frac{\partial u_{i}\left(z_{i}, t\right)}{\partial z}=D_{i+1, z} \frac{\partial u_{i+1}\left(z_{i}, t\right)}{\partial z}, i=N-1, u_{i}(z, 0)=u_{i 0}, i=\overline{1, N},
\end{array}\right.
$$

where $u_{i}=u_{i}(z, t)-$ the concentration functions in every layer;

$q_{i}=r_{i+1 z} / r_{i z}$ - the "jumps" of convection-coefficients;

$F_{i}, C_{0 z}, C_{a z}, D_{i z}, r_{i z}, a_{i 0}$ - the constant coefficients;

$\alpha_{z}, \beta_{z}$ - the constant mass transfer coefficients in the 3-rd kind boundary conditions;

$C_{a z}, C_{0 z}$ - the given concentration on the boundary;

$t_{f}-$ the final time;

$u_{i 0}$ - the given initial condition.

If $N>2$ then the solution is discontinuous [3], if $N=1-$ the conditions on the contact line are deleted.

\subsection{Averaging in z-direction applying integral spline of exponential type $(N=2)$}

In the case of two layers, the spline function is in the form [7]:

$u_{i}(z, t)=\frac{1}{H_{i}} \int_{z_{i-1}}^{z_{i}} u_{i}(z, t) d z+m_{i z}(t) f_{i z 1}+e_{i z}(t) f_{i z 2}, \quad f_{i z 1}=\exp \left(a_{1 i z}\left(z-\bar{z}_{i}\right)\right)-\frac{2}{a_{1 i z}} \operatorname{sh}\left(0.5 a_{1 i z} H_{i}\right)$, 


$$
\begin{gathered}
f_{i z 2}=\exp \left(a_{2 i z}\left(z-\bar{z}_{i}\right)\right)-\frac{2}{a_{2 i z}} \operatorname{sh}\left(0.5 a_{2 i z} H_{i}\right), \int_{z_{i-1}}^{z_{i}} f_{i z 1}(z) d z=\int_{z_{i-1}}^{z_{i}} f_{i z 2}(z) d z=0, \\
\bar{z}_{i}=\left(z_{i-1}+z_{i}\right) / 2, z \in\left[z_{i-1}-z_{i}\right], i=\overline{1,2}
\end{gathered}
$$

Such parameters are used

$$
a_{1 i z}=-\frac{r_{i z}}{2 D_{i z}}-\sqrt{\frac{r_{i z}^{2}}{4 D_{i z}^{2}}+\frac{a_{i 0}^{2}}{D_{i z}}}, a_{2 i z}=-\frac{r_{i z}}{2 D_{i z}}+\sqrt{\frac{r_{i z}^{2}}{4 D_{i z}^{2}}+\frac{a_{i 0}^{2}}{D_{i z}}}, i=\overline{1,2}
$$

These parameters are the characteristic values for the equation $D_{i z} \lambda^{2}+r_{i z} \lambda-a_{i 0}^{2}=0$ corresponding to the ordinary homogenous differential equation $D_{i z} u^{\prime \prime}(z)+r_{i z} u^{\prime}(z)-a_{i 0}^{2}(z)=0$ with the independent variable $z$. The functions $m_{i z}=m_{i z}(t), e_{i z}=e_{i z}(t)$ are found, as in previous publications [2], from boundary conditions of (1.1) at $z_{0}=0, z=z_{1}, z=L_{Z}$, but in addition a condition $q_{i}=r_{i+1 z} / r_{i z}$ that determines the "jump-off" effect of the solution is used.

$$
\begin{aligned}
& m_{1 z}=m_{01}\left(q_{1} u_{2 z}-u_{1 z}\right)+m_{11} e_{1 z}+m_{12} e_{2 z}, m_{2 z}=m_{02}\left(q_{1} u_{2 z}-u_{1 z}\right)+m_{21} e_{1 z}+m_{22} e_{2 z} \\
& m_{01}=D_{2 z} a_{21}^{m} / s_{1}, s_{1}=D_{2 z} a_{21}^{m} d_{11}^{p}-D_{1 z} a_{11}^{p} d_{21}^{m} q_{1}, m_{02}=m_{01} s_{2}, s_{2}=a_{11}^{p} D_{1 z} /\left(a_{21}^{m} D_{2 z}\right), \\
& m_{11}=\left(D_{1 z} a_{12}^{p} d_{21}^{m} q_{1}-D_{2 z} a_{21}^{m} d_{12}^{p}\right) / s_{1}, m_{12}=D_{2 z} q_{1}\left(a_{21}^{m} d_{22}^{m}-a_{22}^{m} d_{21}^{m}\right), \\
& m_{21}=m_{11} s_{2}+a_{12}^{p} D_{1 z} /\left(a_{21}^{m} D_{2 z}\right), m_{22}=m_{12} s_{2}-a_{22}^{m} / a_{21}^{m} . \\
& d_{i k}^{m}=\exp \left(-0.5 a_{k i z} H_{i}\right)-\frac{2}{a_{k i z} H_{i}} \operatorname{sh}\left(0.5 a_{k i z} H_{i}\right), d_{i k}^{p}=\exp \left(0.5 a_{k i z} H_{i}\right)-\frac{2}{a_{k i z} H_{i}} \operatorname{sh}\left(0.5 a_{k i z} H_{i}\right), \\
& a_{i k}^{m}=a_{k i z} \exp \left(-0.5 a_{k i z} H_{i}\right), a_{i k}^{p}=a_{k i z} \exp \left(0.5 a_{k i z} H_{i}\right), i, k=\overline{1,2} . \\
& e_{1 z}=b_{11} u_{1 z}+b_{12} u_{2 z}+g_{3}, e_{2 z}=b_{21} u_{1 z}+b_{22} u_{2 z}+g_{4}, b_{11}=\left(d_{22}\left(\beta_{1}+b_{1}\right)-d_{12} b_{2}\right) / l, \\
& b_{12}=\left(d_{12}\left(\alpha_{1}+b_{2} q_{1}\right)-d_{22} b_{1} q_{1}\right) / l, b_{21}=\left(-d_{12}\left(\beta_{1}+b_{1}\right)+d_{11} b_{2}\right) / l, \\
& b_{22}=\left(-d_{11}\left(\alpha_{1}+b_{2} q_{1}\right)+d_{21} b_{1} q_{1}\right) / l, \beta_{1}=\beta / D_{1 z}, \alpha_{1}=\alpha / D_{2 z}, b_{1}=m_{01}\left(a_{11}^{m}-\beta_{1} d_{11}^{m}\right), \\
& b_{2}=m_{02}\left(a_{21}^{p}+\alpha_{1} d_{21}^{p}\right), g_{3}=-\left(C_{0 z} \beta_{1} d_{22}+C_{a z} \alpha_{1} d_{12}\right) / l, \\
& g_{4}=\left(C_{0 z} \beta_{1} d_{21}+C_{a z} \alpha_{1} d_{11}\right) / l, l=d_{11} d_{22}-d_{12} d_{21}, d_{11}=a_{11}^{m} m_{11}+a_{12}^{m}-\beta_{1}\left(d_{11}^{m} m_{11}+d_{12}^{m}\right), \\
& d_{12}=m_{12}\left(a_{11}^{m} m_{11}-\beta_{1} d_{11}^{m}\right), d_{21}=m_{21}\left(a_{21}^{p}+\alpha_{1} d_{21}^{p}\right), d_{22}=a_{21}^{p} m_{22}+a_{22}^{p}+\alpha_{1}\left(d_{21}^{p} m_{22}+d_{22}^{p}\right) .
\end{aligned}
$$

Applying CAM with respect to IBVP (1.1), we get a system of ODEs for the function to be searched, $u_{i}=u_{i}(z, t), i=1^{-}, 2$;

$$
\begin{aligned}
& \left\{\begin{array}{l}
\dot{u}_{1 z}=c_{11} u_{1 z}+c_{12} u_{2 z}+g_{1}, \\
\dot{u}_{2 z}=c_{21} u_{1 z}+c_{22} u_{2 z}+g_{2}, \\
u_{1 z}(0)=u_{10}, u_{2 z}(0)=u_{20} .
\end{array}\right. \\
& c_{11}=e_{11}\left(-m_{01}+m_{11} b_{11}+m_{12} b_{21}\right)+e_{12} b_{11}-a_{10}^{2}, c_{12}=e_{11}\left(q_{1} m_{01}+m_{11} b_{12}+m_{12} b_{22}\right)+e_{12} b_{12} \text {, } \\
& c_{21}=e_{21}\left(-m_{02}+m_{21} b_{11}+m_{22} b_{21}\right)+e_{22} b_{21}, c_{22}=e_{21}\left(q_{1} m_{02}+m_{21} b_{12}+m_{22} b_{22}\right)+e_{22} b_{22}-a_{20}^{2} \text {. } \\
& e_{11}=\frac{2}{H_{1}} \operatorname{sh}\left(0.5 a_{11 z} H_{1}\right)\left(D_{1 z} a_{11 z}+r_{1 z}\right), e_{12}=\frac{2}{H_{1}} \operatorname{sh}\left(0.5 a_{21 z} H_{1}\right)\left(D_{1 z} a_{21 z}+r_{1 z}\right) \text {, } \\
& e_{21}=\frac{2}{H_{2}} \operatorname{sh}\left(0.5 a_{12 z} H_{2}\right)\left(D_{2 z} a_{12 z}+r_{2 z}\right), e_{22}=\frac{2}{H_{2}} \operatorname{sh}\left(0.5 a_{22 z} H_{2}\right)\left(D_{2 z} a_{22 z}+r_{2 z}\right) \text {, } \\
& g_{1}=e_{11}\left(g_{3} m_{11}+g_{4} m_{12}\right)+e_{12} g_{3}+F_{1}, g_{2}=e_{21}\left(g_{3} m_{21}+g_{4} m_{22}\right)+e_{22} g_{4}+F_{2} \text {. }
\end{aligned}
$$


The solution of the system (1.3) is in the form of vector-matrices:

$$
v(t)=\exp (A \cdot t)\left(v_{0}+A^{-1} \cdot F\right)-A^{-1} \cdot F
$$

with a matrix

$$
A=\left(\begin{array}{ll}
c_{11} & c_{12} \\
c_{21} & c_{22}
\end{array}\right)
$$

and vectors-columns

$$
v(t)=\left[u_{1 z}(t) ; u_{2 z}(t)\right], v_{0}=\left[u_{10} ; u_{20}\right], F=\left[g_{1} ; g_{2}\right] .
$$

The stationary averaged solution of the (1.1) is

$$
\left\{\begin{array}{l}
u_{1 z}=\left(g_{2} c_{12}-g_{1} c_{22}\right) / d, \\
u_{2 z}=\left(-g_{2} c_{11}+g_{1} c_{21}\right) / d, d=c_{11} c_{22}-c_{12} c_{21} .
\end{array}\right.
$$

The stationary analytical solution of the (1.1) is

$$
\left\{\begin{array}{l}
u_{1}(z)=P_{1} \exp \left(a_{11 z} z\right)+P_{2} \exp \left(a_{21 z} z\right)+f_{1}, \\
u_{2}(z)=P_{3} \exp \left(a_{12 z} z\right)+P_{4} \exp \left(a_{22 z} z\right)+f_{2} .
\end{array}\right.
$$

$P_{1}=-\left(b_{1} P_{2}+b_{2}\right), P_{2}=\left(b_{5} b_{22}+b_{6} b_{12}\right) / d, P_{3}=b_{3} P_{4}-b_{4}, P_{4}=\left(b_{6} b_{11}-b_{5} b_{21}\right) / d$,

$d=b_{11} b_{22}-b_{12} b_{21}, b_{1}=\frac{\beta_{1}-a_{21 z}}{\beta_{1}-a_{11 z}}, b_{2}=\frac{\beta_{1}\left(f_{1}-C_{0 z}\right)}{\beta_{1}-a_{11 z}}$,

$b_{3}=-\exp \left(L_{z}\left(a_{22 z}-a_{12 z}\right)\right) \frac{\alpha_{1}+a_{22 z}}{\alpha_{1}+a_{12 z}}, b_{4}=-\exp \left(-L_{z} a_{12 z}\right) \frac{\alpha_{1}\left(f_{2}-C_{a z}\right)}{\alpha_{1}+a_{12 z}}$

$b_{5}=f_{2} q_{1}-f_{1}+b_{2} \exp \left(a_{11 z} H_{1}\right)-q_{1} b_{4} \exp \left(a_{12 z} H_{1}\right)$,

$b_{6}=b_{2} a_{11 z} \exp \left(a_{11 z} H_{1}\right)-b_{4} a_{12 z} k \exp \left(a_{12 z} H_{1}\right), k=D_{2 z} / D_{1 z}$,

$b_{11}=-b_{1} \exp \left(a_{11 z} H_{1}\right)+\exp \left(a_{21 z} H_{1}\right), b_{12}=-q_{1} b_{3} \exp \left(a_{12 z} H_{1}\right)+\exp \left(a_{22 z} H_{1}\right)$,

$b_{21}=-b_{1} a_{11 z} \exp \left(a_{11 z} H_{1}\right)+a_{21 z} \exp \left(a_{21 z} H_{1}\right), b_{22}=-k\left(b_{3} a_{12 z} \exp \left(a_{12 z} H_{1}\right)+a_{22 z} \exp \left(a_{22 z} H_{1}\right)\right)$,

$f_{1}=F_{1} / a_{10}^{2}, f_{2}=F_{2} / a_{20}^{2}$.

\subsection{Averaging in z-direction applying integral spline of hyperbolic type $(N=2)$}

In the case of two layers, the hyperbolic type spline function is in the form [8]:

$u_{i}(z, t)=u_{i z}(t)+m_{i z}(t) f_{i z 1}+G_{i} e_{i z}(t) f_{i z 2}$,

$G_{i}=H_{i} / D_{i z}, f_{i z 1}=0.5 H_{i} \frac{\operatorname{sh}\left(a_{1 i z}\left(z-\bar{z}_{i}\right)\right)}{\operatorname{sh}\left(0.5 a_{1 i z} H_{i}\right)}, f_{i z 2}=0.25\left(\frac{s^{2}\left(a_{2 i z}\left(z-\bar{z}_{i}\right)\right)}{s h^{2}\left(0.5 a_{2 i z} H_{i}\right)}-A_{i 0}\right)$,

$u_{i z}(t)=\frac{1}{H_{i}} \int_{z_{i-1}}^{z_{i}} u_{i}(z, t) d z, A_{i 0}=0.25 \frac{\operatorname{sh}\left(a_{2 i z} H_{i}\right) /\left(a_{2 i z} H_{i}\right)-1}{\operatorname{ch}\left(a_{2 i z} H_{i}\right)-1}, \bar{z}_{i}=\left(z_{i-1}+z_{i}\right) / 2, z \in\left[z_{i-1}-z_{i}\right]$, $i=\overline{1,2}$.

For hyperbolic type functions we use the following parameters (optimal parameters for solving diffusion problem without convection [8]):

$$
a_{11 z}=a_{10} / \sqrt{D_{1 z}}, a_{21 z}=a_{11 z} / 2, a_{12 z}=a_{20} / \sqrt{D_{2 z}}, a_{22 z}=a_{12 z} / 2 \text {. }
$$

Similarly we can obtain the unknown functions $u_{i z}(t), m_{i z}(t), e_{i z}(t)$, [8]. 


\section{Results and discussion}

\section{Numerical results for linear problem}

This chapter deals with solving of the IBVP (1.1) (linear case - diffusion coefficients $D_{i z}$ of (1.1) are constant) using CAM and MATLAB programme "pdepe". For the purposes of this article, the 1-D mathematical model (1.1) has been supplemented by an improved condition of coherence on layer borders: $q_{i}=r_{i+1 z} / r_{i z}$ - the "jump-off" effect of the solution of IBVP. This is a discontinue solution to the equation of IBVP, which is due to discontinued convection coefficients.

This chapter uses the spline function (exponential, hyperbolic, and parabolic) to get an approximated solution. This makes it possible to assess the effectiveness of the use of spline functions as regards the precision of the approximated solution in the different possible cases of "jump-off" effect.

Another literature source [9] also provides solving of the 1-D IBVP with the help of "pdepe" using the grid method. In addition we give some possible cases of the convection coefficient $r_{i z}$ of the IBVP.

The following discrete values are used to create the grid:

$$
\begin{aligned}
& x_{j}=j \cdot h, j=\overline{0, N_{x}}, N_{x} \cdot h=L_{z}=3, t_{n}=n \cdot \tau, n=\overline{0, N_{t}}, N_{t} \cdot \tau=t_{f}=19, N_{t}=100, \\
& N_{x}=60, u_{0}=0, C_{a z}=1, C_{0 z}=5, \alpha_{z}=10^{2}, \beta_{z}=10^{-4} .
\end{aligned}
$$

For two-layer environment we have:

$F_{1}=0.6, F_{2}=-0.4, a_{10}=1.2, a_{20}=0.8, D_{1 z}=10^{-1}, D_{2 z}=10^{-2}, H_{1}=1.8, H_{2}=1.2$.

We use three possible cases of convection coefficients:

1. $r_{1 z}=r_{2 z}=0.1$ (discontinuos derivatives),

2. $r_{1 z}=0.5, r_{2 z}=0.1$ (discontinuos derivatives and solutions),

3. $r_{1 z}=r_{2 z}=0$ (solution without convection).

There are represented the numerical (obtained with "pdepe") and averaged (obtained with CAM for parabolic splines (A. Buikis [1]), exponential type and hyperbolic type splines) for two layers at $r_{1 z}=r_{2 z}=0.1$ in Fig. 1 . The error of approximation for averaged solutions is: with exponential type spline - erexp $=5 \cdot 10^{-9}$; with hyperbolic type spline - erhyp $=0.278$; with parabolic type spline erpar $=0.360$, for numerical - ernum $=0.0119$. There is represented the analytical (exact) solution (1.5) and averaged (1.4) (obtained with CAM) solution at $r_{1 z}=0.5, r_{2 z}=0.1$ in Fig. 2 with the following errors: erexp $=10^{-9}$, erhyp $=0.270$, erpar $=0.326$. In the case $r_{1 z}=r_{2 z}=0$ (solution without convection) the following calculation errors were obtained: $\operatorname{erexp}=2 \cdot 10^{-12}$, $\operatorname{erhyp}=2 \cdot 10^{-12}$, erpar $=0.439$, ernum $=0.0108$.

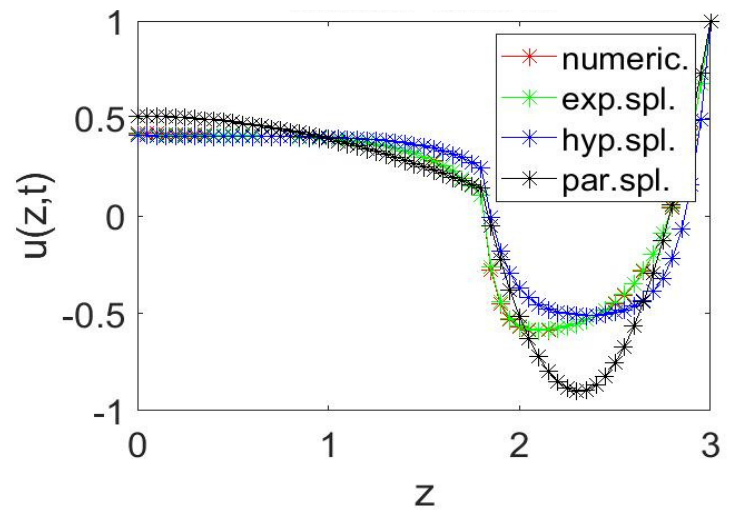

Fig. 1. Nonstationary solution obtained by "pdepe" and CAM at $r_{1 z}=0.5, r_{2 z}=0.1$, $t=t_{\mathrm{f}}$ ernum $=0.0119, \operatorname{erexp}=5 \cdot 10^{-9}$, erhyp $=0.278$, erpar $=0.360$

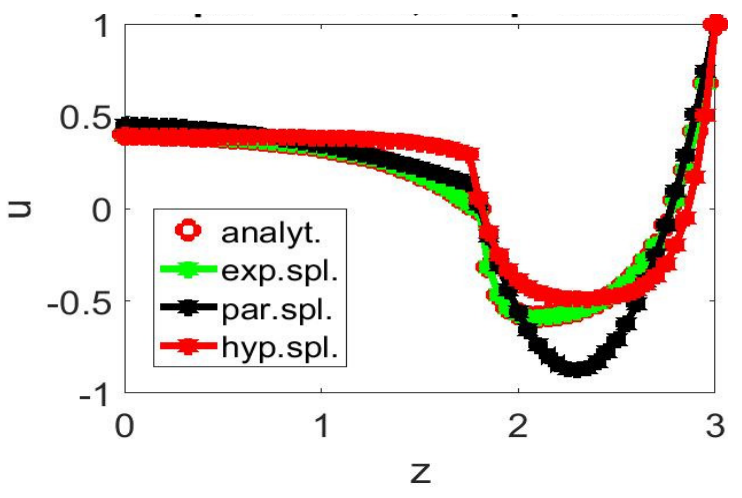

Fig. 2. Stationary solution (analytical and obtained by CAM) at $\boldsymbol{r}_{1 z}=0.5, r_{2 z}=0.1$ erexp $=10^{-9}$, erhyp $=0.270$, erpar $=0.326$ 


\section{Application for modelling of heat transfer problem for two layered gypsum board products exposed to heating}

For the purposes of this chapter we shall use the experimental data obtained on the heating of gypsum plates [10]. In order to determine the coefficients' $c_{p}$ (specific heat) and $K$ (thermal conductivity) dependency on temperature, the cubic spline interpolation was used, which is more accurate compared to the linear dependency used above [10]. With the help of Math Model (1.1) a temperature distribution depending on the variable coordinate $z$ - the thickness of the gypsum plates, at different time $t$ values was obtained.

The gypsum board material with two layered plates in $z$-direction is studied: a foam gypsum plate of a thickness $H_{1}=0.0125 \mathrm{~m}$ and density $\rho_{1}=300 \mathrm{~kg} \cdot \mathrm{m}^{-3}$ and gypsum carton plate $-H_{1}=0.0125 \mathrm{~m}$ and $\rho_{1}=1000 \mathrm{~kg} \cdot \mathrm{m}^{-3}$.

Gypsum plate from the side $z=L_{z}=0.025$ is heated, the functional relationship of temperature is $g(t)=20+345 \cdot \lg (8 t / 60+1){ }^{\circ} \mathrm{C}, t-$ time in minutes [10].

Based on literature sources [2;5] 11, 14 the $c_{p}$ (specific heat) and $K$ (thermal conductivity) dependency on time was found through the cubic spline interpolation, see Fig. 3, 4. In this case we have in equations of the IBVP (1.1) the thermal diffusion coefficients

$$
D_{1 z}=\frac{K}{c_{p} \rho_{1}}, D_{2 z}=\frac{K}{c_{p} \rho_{2}}
$$

depending on the temperature. For the initial condition for $t=0$ we give $u_{1}(z, 0)=u_{0}(z, 0)=u_{0}$, where $u_{0}=20^{\circ} \mathrm{C}$. The following boundary conditions should be applied to the IBVP (1.1) according to the task under consideration for gypsum plates: $r_{1 z}=r_{2 z}=0, r_{i 0}^{2}=-, F_{i}=0, q_{i}=0, \beta_{z}=0, \alpha_{z}=\infty$, $C_{a z}=g(t)$.

The results of calculations are obtained by MATLAB.

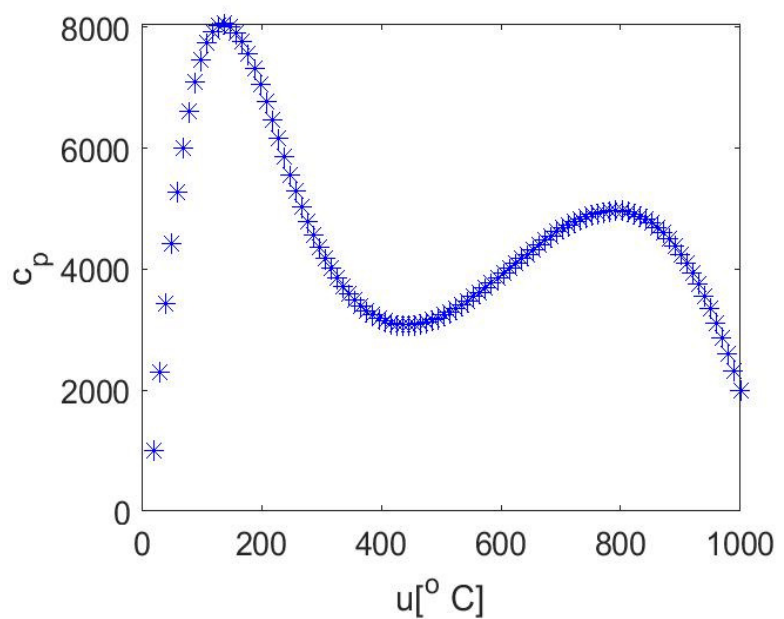

Fig. 3. Specific heat $c_{p}$ dependence on temperature $u$

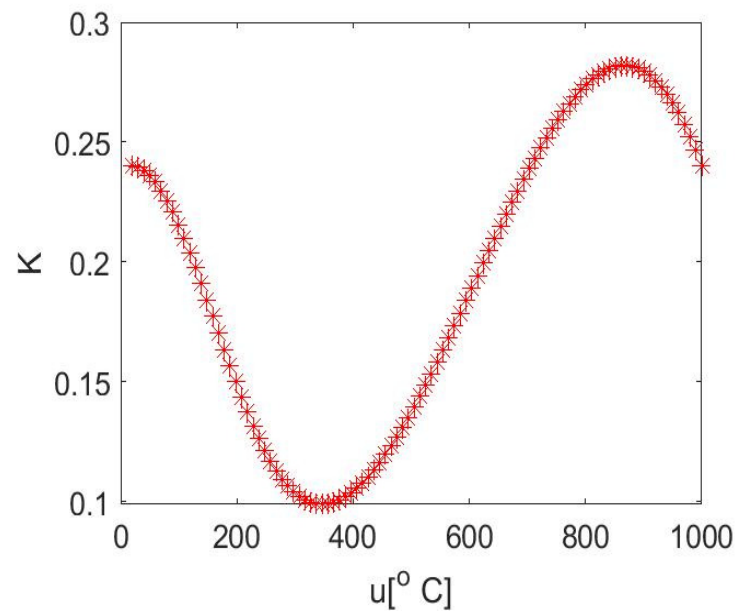

Fig. 4. Thermal conductivity $K$ dependence on temperature $u$

The results for the final time $t_{f}=831 / 3 \mathrm{~min}, L_{z}=0.025, H_{1}=H_{2}=0.0125$ are represented in Fig. $5 \quad$ with $\quad u\left(0, t_{f}\right)=512.5^{\circ} \mathrm{C}, \quad u\left(H_{1}, t_{f}\right)=595.7^{\circ} \mathrm{C}, \quad u\left(L_{z}, t_{f}\right)=994.5^{\circ} \mathrm{C}, \quad u(0,25)=70.2^{\circ} \mathrm{C}$, $u(0,50)=199.9^{\circ} \mathrm{C}$.

Fig. 6 shows very good coincidence between the experimental measurements with the obtained numerical calculations, where [11]:

$u_{2}\left(L_{z}, t\right)=[476,599,701,721,746,761,781,804,815,833,854,861,872,891,902] ;$

$u_{1}(0, t)_{\text {exp }}=[24.4,24.5,40.0,45.7,54.8,56.8,57.3,62.2,69.4,79.2,96.1,102.8,115.2,141.6,147.6]$;

$u_{1}(0, t)_{\text {num }}=[20.6,24.8,35.7,39.3,45.0,49.1,55.6,65.1,70.2,81.3,96.9,103.7,115.0,142.4,164.9] ;$

In the Fig. 6 there are compared the numerical $\left(u_{1}(0, t)_{\text {num }}\right)$ and experimental $\left(u_{1}(0, t)_{\text {exp }}\right)$ results for temperatures $u_{2}\left(L_{z}, t\right)$ in the interval $[476,902]$. 


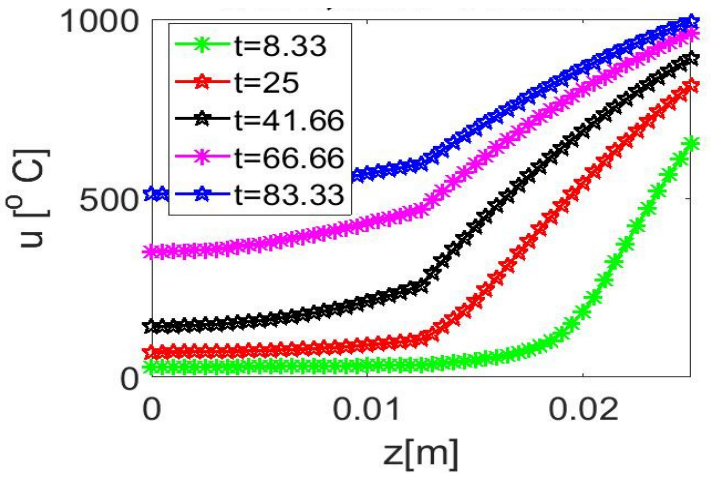

Fig. 5. Solution $u(z, t)$ depending on $z$ at fixed $t$ values, $u_{1}\left(0, t_{f}\right)=\mathbf{5 1 2 . 5 2}$

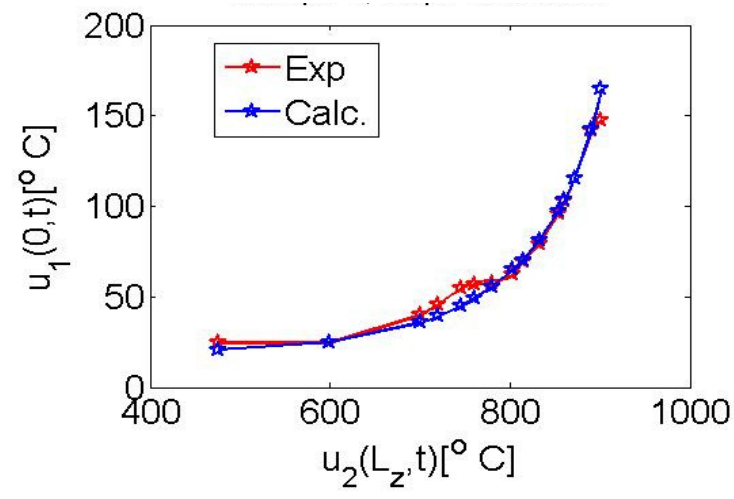

Fig. 6. Comparsion of numerical (Calc) and experimental (Exp) results

Using the Paired-Comparison test [7]:" $t$-Test Paired Two Sample for Means" with MS Excel between the values $u_{1}(0, t)_{\text {exp }}$ and $u_{1}(0, t)_{\text {num }}$, the results of

0.332 ( $t$ Stat $)<2.145$ ( $t$ Critical two-tail) $(\alpha=0.05$ - level of significance) indicate that the difference between the values to be checked $\left(u_{1}(0, t)_{\exp }\right.$ and $\left.u_{1}(0, t)_{\text {num }}\right)$ is not statistically significant [7], which can be interpreted as a good coincidence of the experimental and corresponding predicted numerical results.

A similar result at matching the pairs of $u_{1}(0, t)_{\exp }$ and $u_{1}(0, t)_{n u m}$ was also obtained with IBM SPSS Statistics20, comparing the above mentioned data pairs with the "Wilcoxson Signed Ranks Test" [12]: 0.649 (asymp. Sig. 2-tailed) $>0.050$ (level of significance).

In addition to the statistical tests performed, the calculated square error (SQE) between the experimental measurements and the obtained numerical calculations was $\mathrm{SQE}=1.6077$ ( $\mathrm{SQE}=2.2 \%$ calculated as a percentage),

$$
S Q E=\sqrt{\frac{\sum_{i}\left(\bar{d}_{i}-d_{i}\right)^{2}}{n \cdot(n-1)}},
$$

where $d_{i}$-difference between the experimental measurements and the numerical calculations; $\bar{d}_{i}-$ average difference of the measurements concerned.

\section{Conclusions}

1. The investigational nonstationary diffusion-convection initial-boundary value problem (IBVP) in multi-layer environment is being transformed to the initial value problem of ordinary differential equations due to the designed spline function's varieties - the hyperbolic and the exponential.

2. A stationary analytical solution was obtained in the solving of the 1-D IBVP, the use of CAM resulted in a nonstationary solution and stationary averaged solution. Applying the Matlab "pdepe", the numerical results in solving linear and nonlinear 1-D IBVP were obtained.

3. Solving the 1-D diffusion - convection IBVP resulted in a "jump-off" effect of the obtained solution. Calculations with CAM using exponential type splines showed several times higher accuracy than with Matlab "pdepe" - this indicates the effectiveness of using CAM.

4. Studying the effectiveness of CAM, also solving the 1-D diffusion IBVP, it was found that the accuracy of the solution obtained, both by hyperbolic and exponential type splines, was higher than that of obtained by Matlab "pdepe" programme.

5. The statistic method used - Paired-Comparison test with MS Excel and IBM SPSS Statistics20 allowed for comparison of the experimental results with the numerical results obtained by solving the system of PDEs and thus enabled quantitative characterisation of the good coincidence of the comparable data, which in turn gave a qualitative characterisation of the mathematical model created - its usefulness for studying heat transfer processes of gypsum products exposed to fire. 


\section{Acknowledgments}

This work was partially supported by the European Regional Funding for the project SAM 1.1.1.1./16/A/004

\section{References}

[1] Buikis A. The approximation with splines for problems in layered systems. Riga, Acta Universitatis Latviensis, 592 1994, pp. 135-138 (In Latvian).

[2] Buikis A., Kalis H., Kangro I. Special Splines of Exponential Type for the Solutions of Mass Transfer Problems in Multilayer Domains. Mathematical Modelling and Analysis, vol. 21, issue 4, 2016, pp. 450-465.

[3] Tsurko V.A. Finite-difference methods for convection-diffusion problems with discontinuous coefficients and solutions. Differential Equations, 41: 2, 2005, pp. 290-296.

[4] Mydin O., Awang H.A. Review on properties of gypsum plasterboards after exposure to high temperatures Proceedings of the International conference "International Building \& Infrastructure Technology Conference", 2014, pp. 409-415.

[5] Neuberger P., Aboltins A., Kic P. Effect of high temperatures on gypsum-based composites. Proceedings of International conference "17th International conference Engineering for Rural Development", May 23-25, 2018, Jelgava, Latvia, vol. 17, 2018, pp. 1624-1627.

[6] Qingfeng $\mathrm{Xu}$, Yong Wang, Lingzhu Chen, Rundong Gao, Xiangmin Li. Comparative experimental study of fire-resistance ratings of timber assemblies with different fire protection measures. Advances in Structural Engineering, 2016, Vol. 19(3), pp. 500-512.

[7] Arhipova I., Bāliņa S. Statistika ar Excel ikvienam. Mācību līdzeklis. 1., 2. dạ̣a. Rīga, Datorzinību centrs, 2000.

[8] Kalis H., Buiķis A., Aboltins A., Kangro I. Special Splines of Hyperbolic Type for the Solutions of Heat and Mass Transfer 3-D Problems in Porous Multi-Layered Axial Symmetry Domain. Mathematical Modelling and Analysis, vol. 22, issue 04, 2017, pp. 425-440. Elsevier SCOPUS.

[9] Thomas J.W. Numerical partial differential equations. Finite difference methods. New-York: Springer-Verlag, Inc., 1995.

[10] Aboltins A., Kalis H., Pulkis K., Skujans J., Kangro I. On Mathematical Modelling of Heat Transfer problem for a two layered gypsum board products exposed to fire. Proceedings of International conference "16th International Scientific Conference ENGINEERING FOR RURAL DEVELOPMENT”, May 24-26, 2017, Jelgava, Latvia, vol. 16, 2017, pp. 1369-1376.

[11] Aboltins A., Kalis H., Pulkis K., Skujans J. Numerical simulation of heat transfer problem for layered gypsum products exposed to fire. Proceedings of International conference " 17 th International conference Engineering for Rural Development", May 23-25, 2018, Jelgava, Latvia, vol. 17, 2018, pp. 1282-1288.

[12] Norušis M. J. SPSS Guide to Data Analysis. Marketing Department SPSS, Inc. 444 North Michigan Avenue Chicago, IL 60611, 1993. 